\title{
Berubicin Hydrochloride
}

National Cancer Institute

\section{Source}

National Cancer Institute. Berubicin Hydrochloride. NCI Thesaurus. Code C61568.

The hydrochloride salt of the anthracycline derivative berubicin with potential antineoplastic activity. Berubicin intercalates into DNA and interrupts topoisomerase II activity, resulting in the inhibition of DNA replication and repair, and RNA and protein synthesis. Unlike other anthracycline derivatives, this agent crosses the blood-brain barrier (BBB). 